\title{
Developing Deaf Students Fraction Skills Requires Understanding Magnitude and Whole Number Division
}

\author{
Keith Mousley ${ }^{1} \&$ Ronald R. Kelly ${ }^{1}$ \\ ${ }^{1}$ National Technical Institute for the Deaf, Rochester Institute of Technology, Rochester, NY, USA \\ Correspondence: Keith Mousley, National Technical Institute for the Deaf, Rochester Institute of Technology, \\ Rochester, NY, USA. E-mail: kxmntm@rit.edu
}

Received: November 11, 2017

Accepted: December 7, 2017 Online Published: December 21, 2017

doi:10.5539/jel.v7n2p12

URL: http://doi.org/10.5539/jel.v7n2p12

\begin{abstract}
Research has shown that fraction magnitude and whole number division are important precursors to learning and understanding fractions. Deaf and hard-of-hearing (DHH) students are consistently challenged with learning fractions from K-12 through college. Sixty DHH college students were tested for both their understanding of magnitude between two fractions and their ability to calculate whole number division. The results showed that both understanding the magnitude between two fractions and whole number division are significantly associated with accurately calculating arithmetic functions of fractions with like denominators and different denominators that required them to add, subtract, multiply, and divide two fractions. Understanding fraction magnitude and whole number division were also significantly associated with their self-rated confidence of math performance with fractions. Tangentially, DHH college students' English reading ability was significantly, but modestly associated with their fraction performance.
\end{abstract}

Keywords: deaf, fractions, long division, fraction magnitude, mathematics

\section{Introduction}

\subsection{Introduce the Problem}

Early mathematical knowledge and skills especially in the area of fractions and whole number division are extremely important to know in order to survive upper level mathematics courses (Seigler et al., 2012). Fraction skills are a major part of mathematics to be mastered and become the road to many occupations like nursing, carpentry, and auto mechanics (Tian \& Siegler, 2016). Research shows that young students who fall behind with fractions and whole number division skills will stay behind throughout high school (Seigler et al., 2012). Understanding fractions is an essential skill to have if one wants to succeed in math at higher levels including calculus (Seigler et al., 2011; Bailey, Seigler, \& Geary, 2014). "With the proper infusion of precise definitions, clear explanations, and symbolic computations, the teaching of fractions can eventually contribute to mathematics learning in general and the learning of algebra in particular" (Wu, 2001). In addition, fractions are necessary for learning in the physical, biological, and social sciences (Hoyles, Noss, \& Pozzi, 2001). Since fraction skills are critically important for students, it is time to have serious discussions with math educators on how to improve the teaching and learning of fractions. For example, "On the 1978 National Assessment of Educational Progress (NAEP), when asked to choose the closest to the sum of 7/8 +12/13 from the list of 1, 2,19, 21 and "don't know," the proportion of correct answers among eighth graders was 24\%" (Carpenter, Corbitt, Kepner, Linquist, \& Reys, 1980). Thomas (2010) examined the relationship between fractions and a first year 9th grade algebra course. She administered a fraction pretest that measures procedural and conceptual fraction knowledge to 107 9th graders enrolled in her Algebra 1 classes. The results showed that $80 \%$ of the students scored below $50 \%$ on the fraction pretest, indicating that they had poor understanding of fraction skills. Students' understanding of fractions is a continuing challenge.

Deaf and hard-of-hearing (DHH) students are equally challenged with learning fractions. To date, there is very little research on DHH students' learning of fractions including ratio, decimal, and percentage. Co-author Keith Mousley began researching fractions around 2006 with the focus on why many DHH college students have a strong dislike for fractions and have not mastered fraction skills by the time they entered college. Based on interviews with DHH college students, Mousley \& Kurz (2015) conducted a study with DHH students, ages 8 to 16 years old that showed they performed at $40 \%$ accuracy for identifying which fractions were larger or smaller. 
Furthermore, these students had difficulty in ranking fractions in order of magnitude; ranking fractions using pictures resulted in $7 \%$ accuracy, while using word descriptions resulted in $21 \%$ accuracy. These findings suggest that young DHH students who have some fluency of English reading may generally have a better understanding of fractions. This study raises many questions and opens the door to further research of DHH students and the challenges they face in learning fractions.

\subsection{Contributions of Whole Number Division to Learning Fractions}

Seigler and Pyke (2013) explain why the relation of whole number division to fractions should be obvious. First, "any fraction can be viewed as a division problem (N/M); in this sense, fractions are division" (p. 1996). Additionally, there are several other reasons why whole number division might be important to understanding fractions:

Alone among whole number operations, division often yields fractional answers (e.g., $14 \div 3=4 \frac{2}{3}$ ). Experience with whole number division also could provide knowledge of the magnitude of some fractions (e.g., 8/2) and a rough sense of the magnitudes of others (e.g., 10/3 is between 3 and 4). In contrast, whole number additions, subtraction, and multiplication never yield fraction answers and thus, do not present opportunitites to learn about them (p. 1996).

Furthermore, research has shown that elementary school students' knowledge of fractions and whole-number division predicts their mathematics achievement in high school and also has a stronger relation to math achievement than any other potential predictor variables (Siegler et al., 2012). Knowledge of fraction magnitudes and arithmetic is correlated for both whole numbers and fractions (Bailey, Seigler, \& Geary, 2014). Developing number sense helps students see how numbers work and relate to each other. For example, developing whole number skills is necessary before learning fractions. Students need to have a clear understanding that 3 is greater than 2 before they understand $3 / 4$ is greater than $1 / 2$. "Amounts of practice in whole number and fraction arithmetic could be correlated due to children's school programs emphasizing arithmetic practice" (Bailey, Seigler, \& Geary, 2014).

In any learning setting, it is important to see the different kinds of activities. One kind of activity is rote practice. In many cases, students may memorize and then forget. Lack of comprehension could hinder the learning of fractions. Several research studies have brought up conceptual and procedural knowledge because both are essential to understanding whole numbers and fractions (Hiebert \& LeFevre, 1986; Rittle-Johnson \& Siegler, 1998). Numerical development clearly includes many important acquisitions other than knowledge of numerical magnitudes, such as learning to count and to solve arithmetic problems (Siegler, Thompson, \& Schneider, 2012). This is a major challenge for all math educators, but especially for educators of DHH students.

\subsection{Research Questions}

There are four research questions for this study of DHH college students using a modified methodology based on the research of Siegler \& Pyke (2013).

1) Is there an association between understanding magnitude of fraction and fraction skills?

2) Is there an association between long-division skills and fraction skills?

3) What is the confidence level of the DHH participants based on their understanding of fraction magnitude and whole number division for calculating each part of this math test consisting of fractions and whole number division?

4) Is there an association between reading literacy and math performance with fractions?

\section{Method}

\subsection{Participants}

The research participants were 60 DHH college students enrolled in mathematics courses at the National Technical Institute for the Deaf (NTID). Previous mathematical research with DHH college students suggests a large effect size for math problem solving (Kelly \& Mousley, 2001; Kelly, Lang, Mousley, \& Davis, 2003; Kelly, 2008), Cohen's (1977) sample size table for a three-group design to obtain power of .80 with a large effect requires a minimum of $n=21$ per group, and for two group comparisons a minimum per group of $n=26$. The analyses presented in this research meet both of these requirements. While recruitment of participants resulted in slight unequal $\mathrm{n}$ per group, analysis of variance is sufficiently robust to violations of the assumption of equal $\mathrm{n}$, provided the size of groups is reasonably similar (Stevens, 1996). No adjustments to the data were made. 
The NTID/RIT Institutional Review Board approved this study and data collection occurred during the 2014/2015 academic year. The DHH participants were recruited from both basic and advanced NTID algebra courses to assure that a range of math skills were represented in this study. Student participants were paid \$20 USD for their participation in this research. Table 1 shows how the 60 participants were organized into three groups based on their ability to correctly assess the magnitude of fractions. Table 2 shows the relevant demographic information for the three groups organized by correct magnitude comparsion performance.

Table 1. Three groups based on correct performance comparing magnitude between two fractions

\begin{tabular}{lll}
\hline Group Status & Number Per Group & Performance Percentage Correct Range \\
\hline Low & 22 & $12.5 \%$ to $50 \%$ \\
Middle & 18 & $56.3 \%$ to $81.3 \%$ \\
High & 20 & $93.8 \%$ to $100 \%$ \\
\hline
\end{tabular}

Table 2. Demographic information for three groups based on magnitude comparison performance

\begin{tabular}{lllll}
\hline \multicolumn{5}{l}{ Fraction Magnitude Performance } \\
\hline Demographics & Low & Middle & High & Significance \\
\hline Age & $21.2(3.4)$ & $20.6(2.5)$ & $20.7(3.1)$ & n.s. \\
ACT Math Score & $16.5(1.9)$ & $17.4(2.2)$ & $20.1(5.2)$ & High $>$ Low, $p<.005$ \\
& & & & Other comparisons $=$ n.s. \\
NTID Reading Score & $100.4(18.1)$ & $119.0(22.5)$ & $112.1(38.9)$ & n.s. \\
\hline
\end{tabular}

Table 3 shows how the same 60 participants were organized into two groups based on their ability to correctly solve whole number division problems. Table 4 shows the demographic information for the two groups organized by long division performance.

Table 3. Two groups organized based on performance with long division of whole numbers

\begin{tabular}{lll}
\hline Group Status & Number Per Group & Performance Percentage Correct Range \\
\hline Low & 28 & $13.3 \%$ to $66.7 \%$ \\
High & 32 & $80 \%$ to $100 \%$ \\
\hline
\end{tabular}

Table 4. Demographic information for two groups based on long division performance

\begin{tabular}{llll}
\hline & Long Division Performance & & \\
\hline Demographics & Low & High & Significance \\
\hline Age & $21.3(3.4)$ & $20.6(2.6)$ & n.s. \\
ACT Math Score & $17.1(3.5)$ & $18.6(3.6)$ & n.s. \\
NTID Reading Score & $113.8(27.6)$ & $105.9(28.1)$ & n.s. \\
\hline
\end{tabular}

\subsection{Materials}

The math test used to address the research questions consisted of 71 total mathematical problems divided into four parts typical of fraction and long division problems in math classes. Examples of each set of mathematical problems as they were presented to the DHH college student participants in the math test are provided below:

1). Magnitude comparison between two fractions $=16$ items.

DECIDE which one is larger $(>)$, smaller $(<)$ or equal to $(=)$.

For example: $\frac{1}{2}<\frac{3}{4}$

$$
\frac{3}{5}-\frac{2}{7}
$$$$
\frac{3}{5}-\frac{5}{11} \quad \frac{3}{5}-\frac{1}{3}
$$$$
\frac{3}{5}-\frac{4}{7}
$$

2). Arithmetic operations of two fractions with the same denominators $=20$ items.

Do NOT use your calculator. Perform the operations. Show all your work. Reduce if needed. 


$$
\frac{3}{4}+\frac{1}{4}=\quad \frac{2}{3}-\frac{1}{3}=\quad \frac{5}{6} * \frac{1}{6}=\quad \frac{1}{8} \div \frac{3}{8}=
$$

3). Arithmetic operations of two fractions with different denominators $=20$ items.

Do NOT use your calculator. Perform the operations. Show all your work. Reduce if needed.

$$
\frac{1}{3}+\frac{2}{5}=\quad \frac{5}{6}-\frac{1}{4}=\quad \frac{5}{6} * \frac{2}{5}=\quad \frac{2}{7} \div \frac{3}{8}=
$$

4). Whole Number Division $=15$ items.

Do NOT use your calculator. Divide the number by hand. Show all your work.

$$
56 \div 8=\quad 306 \div 9=\quad 91 \div 4=\quad 25 \div 8=
$$

After each set of problems, students were asked to rate their confidence on a 10-point Likert scale with the appropriate description inserted into the blank (fraction magnitude; fractions with like denominators, fractions with different denominators, or long division):

How confident are you for doing these type of problems?

$\begin{array}{llllllllll}1 & 2 & 3 & 4 & 5 & 6 & 7 & 8 & 9 & 10 \\ \text { not sure } & & & & & & & & & \text { really sure }\end{array}$

Test reliability was calculated using Cronbach's alpha $(\alpha)$ for internal consistency for the following: fraction assessment $(\alpha=.75)$; total test assessment $(\alpha=.83)$; and confidence assessments $(\alpha=.83)$. Nunnally (1978) recommends a minimum level of .7 for Cronbach's alpha, while Pallant (2013, p. 104) states values above .8 are preferable, and Salkind (, 2014, p. 119) indicates reliability coefficients should be as large as possible between .00 and +1.00 .

\section{Results}

\subsection{Research Question 1}

To examine whether there is an association between DHH college students' understanding of magnitude of fractions and fraction skills, a mixed between-within subjects ANOVA was conducted to assess any differences between the three groups based on those who scored low, middle, and high on the 16-item test of magnitude comparison and their performance on two 20 -item tests consisting of like denominators and different denominators that required them to add, subtract, multiply, and divide two fractions. There was no significant interaction between group and performance with like or different denominators, Wilks' Lambda $=.93, F(1,57)$ $=2.11, p=.13, \eta \mathrm{p}^{2}=.07$, observed power $=.42$. There was a significant main effect for the within subject math performance on like versus different denominators, Wilks' Lambda $=.78, F(1,57)=15.85, p=.0005, \eta \mathrm{p}^{2}$ $=.22$, observed power $=.98$. In addition, the main effect comparing the three groups' (low, middle, high on fraction magnitude test) performance was significant, $F(2,57)=25.40, p=.0005, \eta \mathrm{p}^{2}=.47$, observed power $=$ 1.00 , indicating that one's understanding of fraction magnitude does influence one's performance in calculating arithmetic functions on fractions with like and different denominators. The Bonferroni method for conducting pair-wise multiple comparisons showed that the Low versus Middle groups were not significantly different in performance $\left(\mathrm{M}_{\text {diff }}=2.18, p=.25, C I_{95}=[-5.23 ; .87] ; S E=1.24\right)$. However, the High fraction magnitude group performed significantly better than both the Low group $\left(\mathrm{M}_{\text {diff }}=8.33, p=.0005, C I_{95}=[5.37 ; .11 .29] ; S E=1.20\right)$ and the Middle group $\left(\mathrm{M}_{\mathrm{diff}}=6.15, p=.0005, C I_{95}=[3.04 ; 9.26] ; S E=1.26\right)$. Table 5 provides the means and standard deviations for the three fraction magnitude groups' performances calculating arithmetic functions on two fractions with like versus different denominators.

Table 5. Fraction magnitude groups' arithmetic performance on fractions with like vs. different denominators

\begin{tabular}{llll}
\hline Fraction Magnitude Groups & Mean (SD) & Mean (SD) & $\mathrm{N}$ \\
& Like Denominators & Different Denominators & \\
\hline Low & $8.82(4.3)$ & $5.32(4.3)$ & 22 \\
Middle & $10.39(3.5)$ & $8.11(6.4)$ & 18 \\
High & $15.80(3.6)$ & $15.00(4.2)$ & 20 \\
\hline
\end{tabular}




\subsection{Research Question 2}

To examine whether there is an association between DHH college students' whole number division performance and fraction skills, a mixed between-within subjects ANOVA was conducted to assess any differences between the two groups' (High $>80 \%$ corrrect; Low $<67 \%$ corrrect on 15 -item long division test of whole numbers) performances on the two 20 -item tests consisting of like denominators and different denominators that required them to add, subtract, multiply, and divide two fractions. There was no significant interaction between group and performance with like or different denominators, Wilks' Lambda $=1.0, F(1,58)=2.11, p=.88, \eta \mathrm{p}^{2}=.00$, observed power $=.05$. There was a significant main effect for the within subject math performance on fractions with like versus different denominators, Wilks' Lambda $=.79, F(1,58)=15.70, p=.0005, \eta \mathrm{p}^{2}=.21$, observed power $=.97$ (Like Denominator $M=11.5, C I_{95}=[10.3 ; 12.6], S E=.57$; Different Denominator $M=9.2, C_{95}=$ [7.7; 10.8], $S E=.79$ ). Also, the main effect comparing the two groups' (low and high on test of long division with whole numbers) performance was significant, $F(1,58)=10.68, p=.002, \eta \mathrm{p}^{2}=.16$, observed power $=.90$ (Low $M=8.3, C I_{95}=$ [6.5; 10.1], $S E=.92 ; \operatorname{High} M=12.4, C I_{95}=[10.7 ; 14.2], S E=.86$ ), indicating that one's whole number division skills does influence one's performance in calculating arithmetic functions on fractions with like and different denominators. Table 6 provides the means and standard deviations for the two low and high long division groups' performances for calculating arithmetic functions on two fractions with like versus different denominators.

Table 6. Long division groups' arithmetic performance on fractions with like vs. different denominators

\begin{tabular}{llll}
\hline Long Division Groups & Mean (SD) & Mean (SD) & $\mathrm{N}$ \\
& Like Denominators & Different Denominators & \\
\hline Low & $9.46(3.4)$ & $7.14(5.9)$ & 28 \\
High & $13.50(5.1)$ & $11.34(6.3)$ & 32 \\
\hline
\end{tabular}

\subsection{Research Question 3}

To examine the confidence level of the three groups of DHH college students based on their understanding of magnitude of fractions, a $3 \times 4$ mixed between-within subjects ANOVA was conducted to assess any differences between the three groups for performing with each of the four parts/sets of the math test (fraction magnitude, like denominator fractions, different denominator fractions, and whole number division). There was no significant interaction between group and confidence self-ratings for calculating each of the four test sets, Wilks' Lambda $=.85, F(6,110)=1.55, p=.17, \eta \mathrm{p}^{2}=.08$, observed power $=.58$. There was a significant main effect for the four confidence ratings, Wilks' Lambda $=.57, F(3,55)=13.60, p=.0005, \eta \mathrm{p}^{2}=.43$, observed power $=1.00$. In addition, the main effect comparing the three groups' (low, middle, high on understanding fraction magnitude) confidence levels was significant, $F(2,57)=12.27, p=.0005, \eta \mathrm{p}^{2}=.30$, observed power $=.99$, indicating that one's understanding of fraction magnitude does influence one's confidence levels on all four parts/sets of the math test. Bonferroni multiple comparisons were Low versus Middle $\left(\mathrm{M}_{\text {diff }}=.13, p=1.0, C I_{95}=[-1.31 ; 1.56]\right.$; $S E=.58)$, High versus Low $\left(\mathrm{M}_{\text {diff }}=2.42, p=.0005, C I_{95}=[1.03 ; 3.81] ; S E=.56\right)$, and High versus Middle $\left(\mathrm{M}_{\text {diff }}=2.54, p=.0005, C I_{95}=[1.08 ; 4.01] ; S E=.59\right)$. Table 7 presents the means and standard deviations for each group's confidence levels per the four sets of problems.

Table 7. Confidence means (SD) of low, middle, and high fraction magnitude groups per problem sets

\begin{tabular}{lllll}
\hline Fraction Magnitude Groups & $\begin{array}{l}\text { Fraction } \\
\text { Magnitude }\end{array}$ & Like Denominator & $\begin{array}{l}\text { Different } \\
\text { Denominator }\end{array}$ & Long Division \\
\hline Low $=22$ & $5.36(2.2)$ & $6.36(2.5)$ & $4.64(2.5)$ & $6.36(3.1)$ \\
Middle $=18$ & $5.17(2.2)$ & $6.39(2.4)$ & $4.67(2.4)$ & $6.00(2.8)$ \\
High $=20$ & $8.2(1.9)$ & $8.00(2.2)$ & $7.5(2.4)$ & $8.7(1.8)$ \\
\hline
\end{tabular}

To examine the confidence level of the two groups of DHH college students based on their performance calculating long division of whole numbers, a 2 x 4 mixed between-within subjects ANOVA was conducted to assess any differences between the two groups (low versus high for calculating whole number division) for performing with each of the four parts/sets of the math test (fraction magnitude, like denominator fractions, different denominator fractions, and whole number division). There was a significant interaction between group and confidence self-ratings for calculating each of the four test sets, Wilks' Lambda $=.83, F(3,56)=3.89, p$ 
$=.014, \eta \mathrm{p}^{2}=.17$, observed power $=.80$. There was a significant main effect for the four confidence ratings, Wilks' Lambda $=.60, F(3,56)=12.57, p=.0005, \eta \mathrm{p}^{2}=.40$, observed power $=1.00$. In addition, the main effect comparing the two groups' (low versus, high on whole number division) confidence levels was significant, $F(1,58)=14.95, p=.0005, \eta^{2}=.21$, observed power $=.97$, indicating that one's skill in calculating whole number division does influence one's confidence levels on all four parts/sets of the math test, (Low $\mathrm{M}=5.42$, $\left.C I_{95}=[-4.69 ; 6.15] ; S E=.37\right) ;$ High $\left.\mathrm{M}=7.35, C I_{95}=[6.67 ; 8.04] ; S E=.34\right)$. Table 8 presents the means and standard deviations for the confidence levels on a 10-point Likert rating scale.

Table 8. Confidence means (SD) of low versus high long division groups per problem sets

\begin{tabular}{lllll}
\hline Long Division Groups & $\begin{array}{l}\text { Fraction } \\
\text { Magnitude }\end{array}$ & Like Denominator & $\begin{array}{l}\text { Different } \\
\text { Denominator }\end{array}$ & $\begin{array}{l}\text { Long Division } \\
\text { Whole Numbers }\end{array}$ \\
\hline Low $=28$ & $5.75(2.4)$ & $6.14(2.6)$ & $4.43(2.5)$ & $5.36(2.8)$ \\
High $=32$ & $6.69(2.5)$ & $7.59(2.2)$ & $6.63(2.6)$ & $8.50(2.0)$ \\
\hline
\end{tabular}

\subsection{Research Question 4}

To examine whether there was an association with reading literacy and fraction math performance, the sixty DHH participants were grouped into Lower, Middle, and Higher reading levels relative to their NTID Reading Placement Test scores administered to all incoming NTID students. The students' scores on this 200-point test were categorized as follows: Lower reading levels scored in the range of 0 to 99; Middle reading levels scored in the range of 100 to 119; and Higher reading levels scored in the range of 120 to 172.

A mixed between-within subjects ANOVA was conducted to assess any differences between the three reading level groups and their performance on the three sets of fraction tests: magnitude, like denominators, and different denominators. There was no significant interaction between group and performance with the three sets of fractions tests, Wilks' Lambda $=.98, F(4,112)=.23, p=.92, \eta \mathrm{p}^{2}=.01$, observed power $=.10$. There was a significant main effect for the within subject math performance on the three sets of fraction tests, Wilks' Lambda $=.78, F(2,56)=7.81, p=.001, \eta \mathrm{p}^{2}=.22$, observed power $=.94$. In addition, the main effect comparing the three reading level groups' performance was significant, $F(2,57)=4.66, p=.013, \eta \mathrm{p}^{2}=.14$, observed power $=.76$, suggesting that DHH college students' English reading ability does have an association with their performance in calculating magnitude and arithmetic functions on fractions with like and different denominators. The Bonferroni method for conducting pair-wise multiple comparisons showed that the Lower versus Middle reading level groups were not significantly different in performance $\left(\mathrm{M}_{\text {diff }}=.27, p=1.00, C I_{95}=[-3.20 ; 3.75]\right.$; $S E=1.41)$. However, the Higher reading level group performed significantly better than either the Lower reading level group $\left(\mathrm{M}_{\text {diff }}=3.33, p=.045, C I_{95}=[.06 ; 6.61] ; S E=1.33\right)$ and the Middle reading level group $\left(\mathrm{M}_{\text {diff }}=3.61, p=.029, C I_{95}=[.28 ; 6.93] ; S E=1.35\right)$. Table 9 provides the means and standard deviations for the three reading level groups' performances calculating magnitude between two fractions and arithmetic functions on two fractions with like versus different denominators.

Table 9. Reading level groups' fraction magnitude and arithmetic performance on fractions with like versus different denominators

\begin{tabular}{lllll}
\hline Reading Level Groups & Mean (SD) & Mean (SD) & Mean (SD) & N \\
& Fraction Magnitude & Like Denominators & Different Denominators & \\
\hline Lower & $9.68(4.8)$ & $10.47(4.8)$ & $8.11(6.1)$ & 19 \\
Middle & $9.78(4.0)$ & $10.17(3.3)$ & $7.50(4.9)$ & 18 \\
Higher & $12.65(3.6)$ & $13.70(5.3)$ & $11.91(7.1)$ & 23 \\
\hline
\end{tabular}

\section{Summary and Discussion}

The math test used in this research to assess the DHH college students' fraction skills and confidence levels had an acceptable level of internal consistency based on Cronbach's alpha $(\alpha)$ : fraction assessment $(\alpha=.75)$; total test assessment $(\alpha=.83)$; and confidence assessments $(\alpha=.83)$. All fractions and long division math problems in this study were typical of problems presented in middle school through high school math classes. 


\subsection{Summary of Findings}

1) Research Question 1: DHH college students' understanding of fraction magnitude was clearly associated with calculating arithmetic functions on fractions with like and different denominators accounting for $47 \%$ of the variance in performance. The high group consistently performed significantly better than either the low or middle groups (see Table 3).

2) Research Question 2: DHH college students' skill in whole number division was also associated with calculating arithmetic functions on fractions with like and different denominators accounting for $16 \%$ of the variance in performance. The high group consistently performed significantly better than the low group (see Table 4).

3) With respect to the within subjects main effect of fractions with like versus different denominators, the fractions with different denominators were significantly more challenging for DHH college students, accounting for $22 \%$ of the variance in performance for research questions 1 and 2 respectively.

4) Research Question 3: With respect to DHH college students' self-rated confidence levels for calculating each of the four parts of the math test (fraction magnitude, like denominator fractions, different denominator fractions, and whole number division):

- The significant difference between the three groups organized by understanding of fraction magnitude accounted for $30 \%$ of the variance in their self-rated confidence, with the high group consistently exhibiting the most confidence (see Table 5).

- The significant difference between the two groups organized by long division skills accounted for $21 \%$ of the variance in their self-rated confidence, with the high group consistently exhibiting the most confidence (see Table 6).

5) Research Question 4: DHH college students' English reading level also had a significant but modest association on their calculation performance on the three sets of fraction tests (magnitude, like denominators, and different denominators) accounting for $14 \%$ of the variance in performance. The higher-level reading group consistently performed significantly better than both the lower and middle reading groups (see Table 7).

\subsection{Discussion}

These research findings with deaf students are consistent with other research studies with hearing students. It cannot be emphasized enough that students need to master whole number skills including magnitude and basic operations before getting into any fraction discussion. Understanding the size of fractions is crucial prior to introducing the basic operations because fraction magnitude knowledge promotes fraction arithmetic skill (Fuchs et al., 2013, Bailey, Siegler, \& Geary, 2014). In other words, each student needs to master fraction magnitude before learning fractions operations.

Gabriel (2016, p. 37) describes a number of common errors that both children and adults, make when dealing with fractions:

- $\quad$ applying procedures without understanding the underlying concepts

- wrongly processing numerators and denominators as two separate whole numbers, then applying procedures that are used only with whole numbers resulting in mistakes such as

$\circ \quad$ adding or subtracting fractions (e.g., $1 / 3+1 / 2=2 / 5$ )

- comparing fractions (e.g., $1 / 7>1 / 3$ because 7 is larger than 3 )

- considering fractions as being entities smaller than one

- have difficulties placing fractions on number lines

These examples of common errors apply to DHH students in K-12 through college who struggle with fractions. Students need to continue to focus on the number line for both whole numbers and fractions. They need to really understand the size of the fraction. They also need to understand the function of fractions. The students need to be able to figure out and understand where fractions are located on the number line. Mastering the two-step problem is a necessary skill in order to advance to higher-level math. By mastering whole number division skills without a calculator is a good start. The task of adding or subtracting fractions can wait until long division skills are mastered. Seigler and others have pointed out that students need to continue to work on whole numbers in all four operations before doing fractions. The studies also stress the importance of mastering basic arithmetic before learning fractions which then leads to success in higher level of mathematics. Hiebert \& LeFevre (1986) stressed that teachers should focus on conceptual and procedural mathematical skills especially when doing 
two-step problems or more. One recently study (Gabriel et al., 2013) showed primary school children mastered the part-whole and proportion categories, but struggled to see fractions as numbers, and noted that equivalent fractions are very difficult to understand. This leads to not understanding operational procedures for doing fractions. Gabriel also suggested that more instructional time should be allocated to practice and exercises on procedures.

Recommendations for teachers are:

- Work with number lines with whole numbers then practice magnitude differences of whole numbers.

- Make sure students' understand the difference between $1 / 2$ and $1 / 3$, then work toward more complex fractions. Once that is mastered, place fractions on a number line and practice fraction magnitude.

- Thinking out loud during problem solving (Mousley \& Kelly, 1998) promotes conceptual knowledge.

- Have students talk or sign about the fractions to guide their thinking.

- Expose students to real life experience involving fractions as much as possible.

- Work with fractions with a positive attitude in every course (subject).

- $\quad$ Limit usage of calculators until skill is mastered.

\section{References}

Bailey, D., Siegler, R. S., \& Geary, D. C. (2014). Early predictors of middle school fraction knowledge. Developmental Science, 17(5), 775-785. https://doi.org/10.1111/desc.12155

Carpenter, T. P., Corbitt, M. K., Kepner, H., Lindquist, M. M., \& Reys, R. E. (1980). Results and implications of the Second NAEP Mathematics Assessment: Elementary school. Arithmetic Teacher, 27(8), 10-12, 44-47.

Cohen, J. (1977). Statistical power analysis for the behavioral sciences (revised edition). New York, NY: Academic Press.

Fuchs, L. S., Schumacher, R. F., Long, J., Namkung, J., Hamlett, C. L., Cirino, P. T., ... Changas, P. (2013). Improving at risk learners' understanding of fractions. Journal of Educational Psychology, 105, 683-700. https://doi.org/10.1037/a0032446

Gabriel, F. (2016). Understanding magnitudes to understand fractions. APMC-Australian Primary Mathematics Classroom, 21(2), 36-40.

Gabriel, F., Coche, F., Szucs, D, Carette, V., Rey, B., \& Content, A. (2013). A Componential view of children's difficulties in learning fractions. Frontiers in Psychology, 4, 1-10. https://doi.org/10.3389/fpsyg.2013.00715

Hiebert, J., \& Lefevre, P. (1986). Conceptual and procedural knowledge in mathematics: An introductory analysis. In J. Hiebert (Ed.), Conceptual and procedural knowledge: The case of mathematics (pp. 1-27). Hillsdale, NJ: Lawrence Erlbaum Associates.

Hoyles, C., Noss, R., \& Pozzi, S. (2001). Proportional reasoning in nursing practice. Journal for Research in Mathematics Education, 32(1), 4-27. https://doi.org/10.2307/749619

Kelly, R. R. (2008). Deaf learners and mathematical problem solving. In M. Marschark \& P. Hauser (Eds.), Deaf cognition: Foundations and Outcomes (pp. 226-249). New York: Oxford University Press. https://doi.org/10.1093/acprof:oso/9780195368673.003.0008

Kelly, R. R., Lang, H. G., Mousley, K., \& Davis, S. (2003). Deaf college students' comprehension of relational language in arithmetic compare problems. Journal of Deaf Studies and Deaf Education, 8(2), 120-132. https://doi.org/10.1093/deafed/eng006

Kelly, R. R., \& Mousley, K. (2001). Solving Word Problems: More Than Reading Issues for Deaf Students. American Annals of the Deaf, 146(3), 251-262. https://doi.org/10.1353/aad.2012.0088

Mousley, K., \& Kelly, R. R. (1998). Problem-solving strategies for teaching mathematics to deaf students. American Annals of the Deaf, 143(4), 325-336. https://doi.org/10.1353/aad.2012.0082

Mousley, K., \& Kurz, C. (2015). Pre-college deaf students' understanding of fractional concepts: What we know and what we do not know. Journal of Science Education for Students with Disabilities, 18(1), 38-60. Retrieved from http://scholarworks.rit.edu/jsesd/vol18/iss1/7/

Pallant, J. (2013). SPSS survival manual: A step by step guide to data analysis using IBM SPSS (5th edition). Berkshire, UK: McGraw-Hill Education. 
Rittle-Johnson, B., \& Siegler, R. S. (1998). The relations between conceptual and procedural knowledge in learning mathematics: A review. In C. Donlan (Ed.), The development of mathematics skills (pp. 75-110). Hove, England: Psychology Press.

Salkind, N. J. (2014). Statistics for people who think they hate statistics (5th edition). Thousand Oaks, CA: Sage Publications.

Siegler, R. S., Duncan, G. J., Davis-Kean, P. E., Duckworth, K., Claessens, A., Engel, M., ... Chen, M. (2012). Early predictors of high school mathematics achievement. Psychological Science, 23, 691-697. https://doi.org/10.1177/0956797612440101

Siegler, R. S., \& Pyke, A. A. (2013). Developmental and individual differences in understanding fractions. Developmental Psychology, 49(10), 1994-2004. https://doi.org/10.1037/a0031200

Siegler, R. S., Thompson, C. A., \& Schneider, M. (2011). An integrated theory of whole number and fractions development. Cognitive Psychology, 62, 273-296. https://doi.org/10.1016/j.cogpsych.2011.03.001

Stevens, J. (1996). Applied multivariate statistics for the social sciences (3rd edition). Mahwah, NJ: Lawrence Erlbaum.

Tian, J., \& Siegler, R. S. (2016) Fractions learning in children with mathematics difficulties. Journal of Learning Disabilities, 1-7. https://doi.org/10.1177/0022219416662032

Thomas, C. (2010). Fraction competency and algebra success. Thesis. Louisiana State University and Agricultural and Mechanical College, Baton, Rouge, LA. Retrieved from http://digitalcommons.lsu.edu/gradschool_theses/3578

Wu, H. (2001). How to prepare students for algebra. American Educator, 25(2), 10-17. Retrieved from https://pdfs.semanticscholar.org/f3b1/9fb5c237cd151d16f197c6c60d6521195044.pdf

\section{Copyrights}

Copyright for this article is retained by the author(s), with first publication rights granted to the journal.

This is an open-access article distributed under the terms and conditions of the Creative Commons Attribution license (http://creativecommons.org/licenses/by/4.0/). 